Article

\title{
Eruca sativa Might Influence the Growth, Survival under Simulated Gastrointestinal Conditions and Some Biological Features of Lactobacillus acidophilus, Lactobacillus plantarum and Lactobacillus rhamnosus Strains
}

\section{Florinda Fratianni, Selenia Pepe, Federica Cardinale, Tiziana Granese, Autilia Cozzolino, Raffaele Coppola and Filomena Nazzaro*}

Institute of Food Science, ISA-CNR, Via Roma, 64, 83100 Avellino, Italy;

E-Mails: fratianni@isa.cnr.it (F.F.); selenia.pepe@hotmail.it (S.P.); f.sweetgirl@hotmail.it (F.C.); tizianag46@hotmail.it (T.G.); ilia.cozzolino@hotmail.it (A.C.); coppola@isa.cnr.it (R.C.)

* Author to whom correspondence should be addressed; E-Mail: mena@isa.cnr.it; Tel.: +39-825-299-102; Fax: +39-825-781-585.

External Editor: Charles A. Collyer

Received: 5 August 2014; in revised form: 3 September 2014 / Accepted: 17 September 2014 / Published: 1 October 2014

\begin{abstract}
The growth and viability of three Lactobacillus strains, Lactobacillus acidophilus, Lactobacillus plantarum and Lactobacillus rhamnosus, after their passage through simulated gastric and pancreatic juices were studied as a function of their presence in the growth medium of rocket salad (Eruca sativa). The presence of E. sativa affected some of the biological properties of the strains. For example, L. acidophilus and L. plantarum worked more efficiently in the presence of E. sativa, increasing not only the antioxidant activity of the medium, but also their own antioxidant power and antimicrobial activity; L. rhamnosus was not affected in the same manner. Overall, the presence of vegetables might help to boost, in specific cases, some of the characteristics of lactobacilli, including antioxidant and antimicrobial power.
\end{abstract}

Keywords: probiotics; Eruca sativa; Lactobacillus; antioxidant; antimicrobial activity; lab-on-chip protein profile 


\section{Introduction}

Fresh fruit and vegetables represent a key source of naturally occurring antioxidants. It has been reported that a diet containing plant antioxidants may reduce the risk of several diseases [1], such as cardiovascular diseases and different types of cancer, and can generally limit or prevent damage occurring to cellular components due to oxidation [2]. Among natural antioxidants, polyphenols play a very important role [3]. Many of their beneficial health effects can be ascribed to their antioxidant and chelating abilities that give rise to their ability to transfer electron-free radicals, chelate metal catalysts, activate antioxidant enzymes, reduce $R$-tocopherol radicals and inhibit oxidases [4]. Rocket salad (Eruca sativa Miller) is an annual herb belonging to the Brassicaceae (or Cruciferae) family. It is widely recognized to be an important ingredient for culinary and medical/herbalist purposes. Similarly to many of the other Brassicaceae, Eruca sativa consumption might be associated with a highly significant reduction in cancer [5]. Eruca sativa has been known since ancient times in the Mediterranean area and in Central to Western Asia. At present, it is cultivated in Northern Europe, northern America and India. Leaves of rocket salad are intensely green with notched edges and have a characteristic spicy taste that is affected by environmental conditions. Rocked salad is well-known to be a healthy plant that aids in digestion, acts as a diuretic and protects the liver. In addition, it can exhibit chemo-protective action [6], as well as impart beneficial effects on microbiota, and subsequently, it can limit the production of intestine gas. Its main beneficial properties can be ascribed to the presence of vitamin C and some mineral salts (such as iron, calcium and phosphorous) and other biomolecules, such as polyphenols. There is also some evidence that polyphenols may influence microbiota [7]. In an in vitro study, the isoflavone, genistein, was shown to inhibit the internalization of human Caco-2 and HT-29 cells by intestinal bacteria [8]. Parkar et al. [9] highlighted the potential influence of well-known polyphenols on gut micro-ecology by studying their effects in vitro on the growth of bacteria commonly present in the human GI tract. In particular, they reported the novel effect of polyphenols influencing the adhesion of two representative gut bacteria, Salmonella typhimurium (Gram-negative pathogen) and Lactobacillus rhamnosus GG (Gram-positive probiotic), to cultured human Caco-2 enterocytes. Extensive research into the interactions between phenolic compounds and intestinal bacteria has mainly focused on antimicrobial properties against pathogenic bacteria [10-13] to evaluate their potential effects on the shelf life of foods [14]. The growth of different lactobacilli has been previously investigated using different polyphenol matrices [15-19]; however, to our knowledge, there is no literature reporting the effect of the presence of Eruca sativa extracts on Lactobacillus strains. Therefore, our study aimed to evaluate the behavior of three Lactobacillus strains grown in the presence of Eruca sativa. In particular, the growth, the resistance to a simulated gastro-intestinal transit, polyphenol content and the antioxidant and antimicrobial activities, as well as total protein profiles of bacteria were evaluated.

\section{Results and Discussion}

\subsection{Growth of Bacteria and Resistance to the Simulated Digestion}

Generally, the presence of plant extracts does not affect the growth of microorganisms [20,21], but in some cases, they can benefit from the presence of these bio-components, increasing, 
for example, their survival during gastro-intestinal transit. This effect is influenced by both the type of strain and by the vegetable matrix [22]. Table 1 shows the growth of lactobacilli in the presence and absence of E. sativa, before and after simulated digestion. The data are reported as colony forming units $(\mathrm{cfu}) / \mathrm{mL}$.

Table 1. Viability (expressed as cfu/mL) of L. acidophilus (LA), L. plantarum (LP) and L. rhamnosus (LR) grown in MRS medium (see materials and methods) without (used as the control) or with E. sativa (+E), before the in vitro gastric and pancreatic transit (BGPJ and AGPJ, respectively). The data are the mean ( \pm standard deviation) of three independent experiments.

\begin{tabular}{cccc}
\hline \multirow{2}{*}{$\boldsymbol{T}_{\mathbf{0}} \mathbf{c f u} / \mathbf{m L}$ (SD) } & $\begin{array}{c}\text { T 24 h BGPJ treatment } \\
\mathbf{c f u} / \mathbf{m L} \text { (SD) }\end{array}$ & $\begin{array}{c}\text { T 24 h AGPJ treatment } \\
\mathbf{c f u} / \mathbf{m L} \text { (SD) }\end{array}$ \\
\hline LA & $1.23 \times 10^{2}\left(0.01 \times 10^{2}\right)$ & $1.25 \times 10^{8}\left(0.02 \times 10^{2}\right)$ & $1.12 \times 10^{5}\left(0.03 \times 10^{2}\right)$ \\
LA + E & $1.24 \times 10^{2}\left(0.02 \times 10^{2}\right)$ & $8.15 \times 10^{7}\left(0.04 \times 10^{3}\right)$ & $5.29 \times 10^{6}\left(0.02 \times 10^{3}\right)$ \\
LP & $1.34 \times 10^{2}\left(0.02 \times 10^{2}\right)$ & $1.55 \times 10^{8}\left(0.05 \times 10^{3}\right)$ & $2.49 \times 10^{8}\left(0.03 \times 10^{3}\right)$ \\
LP + E & $1.36 \times 10^{2}\left(0.01 \times 10^{2}\right)$ & $2.46 \times 10^{8}\left(0.03 \times 10^{3}\right)$ & $3.34 \times 10^{8}\left(0.05 \times 10^{3}\right)$ \\
LR & $1.27 \times 10^{2}\left(0.02 \times 10^{2}\right)$ & $1.14 \times 10^{8}\left(0.02 \times 10^{3}\right)$ & $1.91 \times 10^{7}\left(0.03 \times 10^{3}\right)$ \\
LR + E & $1.25 \times 10^{2}\left(0.02 \times 10^{2}\right)$ & $4.43 \times 10^{7}\left(0.05 \times 10^{3}\right)$ & $4.16 \times 10^{6}\left(0.03 \times 10^{3}\right)$ \\
\hline
\end{tabular}

L. acidophilus exhibited just a slightly less effective capability to grow in the presence of $E$. sativa than the control (containing $1.25 \times 10^{8} \mathrm{cfu} / \mathrm{mL}$ ). Additionally, the presence of E. sativa seemed to "protect" the microorganism during the simulated digestive process. The L. acidophilus grown in medium MRS showed a marked decrease of cfu/mL from $1.25 \times 10^{8}$ to $1.12 \times 10^{5}$; however, in the presence of the extract, it exhibited a greater resilience to the adverse simulated environmental conditions, so that its viability changed from $8.15 \times 10^{7} \mathrm{cfu} / \mathrm{mL}$ to $5.29 \times 10^{6} \mathrm{cfu} / \mathrm{mL}$, a value still acceptable in terms of probioticity $\left(1 \times 10^{6}-1 \times 10^{8} \mathrm{cfu} / \mathrm{mL}\right.$, as reported by the FAO in 2001) [23]. L. plantarum was not positively influenced by the presence of polyphenols; however, these increased its capability to resist stress. An opposite behavior was demonstrated by L. rhamnosus, which did not receive a clear benefit from the presence of polyphenols in the culture medium before or after the simulated digestion. The presence of polyphenols seemed to dampen bacterial growth compared with the control, and taking into consideration only this parameter, E. sativa could not affect the growth or the resistance to gastro-intestinal transit in any way.

\subsection{Total Polyphenols and Antioxidant Activity}

Some dietary products have a well-known potential to improve the growth and beneficial activities of lactobacilli [24], for example metabolites, such as glycosides and flavonoids [25,26]. In our experiments, a dosage of total polyphenols was transferred to culture medium, with and without E. sativa, to have two comparison values and to assess whether any changes with respect to the content of polyphenols could be attributed to the presence of lactobacilli. The results shown in Table 2 are expressed in $\mu \mathrm{g} / \mathrm{mL}$, compared with the equivalent weight of gallic acid (GAE) used as a standard. 
Table 2. Total polyphenols present in MRS medium with and without E. sativa, before and after $24 \mathrm{~h}$ of growth of L. acidophilus (LA), L. plantarum (LP) and L. rhamnosus (LR). The data are expressed as $\mu$ g gallic acid equivalent (GAE)/mL and represent the mean ( \pm standard deviation) of three independent experiments.

\begin{tabular}{lcc}
\hline & \multicolumn{2}{c}{ Total polyphenols } \\
\cline { 2 - 3 } & $T=0 \mu \mathrm{g} \mathrm{GAE} / \mathrm{mL}(\mathrm{SD})$ & $T=24 \mathrm{~h} \mu \mathrm{g}$ GAE$/ \mathrm{mL}(\mathrm{SD})$ \\
\hline MRS & $130.36(1.07)$ & - \\
MRS + E.sativa & $230.48(2.21)$ & - \\
LA & - & $139.25(2.55)$ \\
LA + E.sativa & - & $218.14(5.14)$ \\
LP & - & $138.04(2.12)$ \\
LP + E. sativa & - & $143.62(3.01)$ \\
LR & - & $136.06(0.55)$ \\
LR + E. sativa & - & $204.03(1.43)$ \\
\hline
\end{tabular}

At time zero, total polyphenols (TPF) in MRS were $130 \mu \mathrm{g} \mathrm{GAE} / \mathrm{mL}$. The presence of $E$. sativa increased the value by nearly $100 \%(230 \mu \mathrm{g}$ GAE/mL). The growth of L. acidophilus in MRS did not influence the content of the total polyphenols of the medium. A decrease of approximately 5\% (218 $\mu \mathrm{g} / \mathrm{mL}$ versus $230 \mu \mathrm{g} / \mathrm{mL}$, respectively) was detected when the strain grew in MRS broth containing E. sativa. L. rhamnosus exhibited a similar behavior, and a decrease of $10 \%$ was observed when it was grown in the presence of E. sativa. L. plantarum exhibited a totally different behavior when grown in the presence of E. sativa; it apparently "consumed" 38\% of the TPF compared to the unfermented medium (143 $\mu \mathrm{g}$ GAE/mL versus $230 \mu \mathrm{g}$ GAE/mL, respectively). The determination of the antioxidant potential of polyphenols was performed using the DPPH test, both on the medium and on microbial pellets, after $24 \mathrm{~h}$ of fermentation. This analysis was carried out to determine whether the presence of E. sativa could affect the antioxidant activity of the bacteria compared to the standard conditions. The results shown in Table 3 are expressed, with regard to the medium of growth, as EC50, indicating the volume of sample $(\mu \mathrm{L})$ needed to decrease the power of $1 \mathrm{~mL}$ of the stable radical DPPH by $50 \%$ and in the percentage of activity for the bacterial pellet.

Table 3. Antioxidant activity evaluated using a DPPH test of medium and microbial pellets of L. acidophilus (LA), L. plantarum (LP) and L. rhamnosus (LR) grown in MRS or MRS $+E$. sativa. The data are expressed in terms of EC50, the amount (as $\mu \mathrm{L}$ ) of sample needed to decrease the activity of $1 \mathrm{~mL}$ of the stable radical DPPH by $50 \%$. The data represent the mean ( \pm standard deviation) of three independent experiments.

\begin{tabular}{|c|c|c|c|}
\hline & \multicolumn{2}{|c|}{ DDPPH test on growth medium } & \multirow{2}{*}{$\begin{array}{c}\text { DDPPH test on microbial pellet } \\
T=24 \mathrm{~h} \\
\text { Antioxidant power (\%) }\end{array}$} \\
\hline & $\begin{array}{c}T=0 \\
\mathrm{EC}_{50}(\mu \mathrm{L} / \mathrm{mL} \pm \mathrm{SD})\end{array}$ & $\begin{array}{c}T=24 \mathrm{~h} \\
\mathrm{EC}_{50}(\mu \mathrm{L} / \mathrm{mL} \pm \mathrm{SD})\end{array}$ & \\
\hline MRS & $74.73(2.64)$ & & \\
\hline MRS + E.sativa & $49.29(2.37)$ & & \\
\hline LA & & $92.13(5.99)$ & 17.90 \\
\hline LA + E.sativa & & $48.64(1.15)$ & 22.95 \\
\hline LP & & $84.80(6.69)$ & 19.41 \\
\hline LP + E.sativa & & $63.98(1.98)$ & 24.02 \\
\hline $\mathrm{LR}$ & & $70.38(2.53)$ & 15.23 \\
\hline LR + E.sativa & & $51.14(2.39)$ & 14.03 \\
\hline
\end{tabular}


The presence of E. sativa obviously increased the antioxidant activity of the medium compared to the control: $25 \mu \mathrm{L}$ less $(49.29 \mu \mathrm{L}$ versus $74.73 \mu \mathrm{L})$ of the culture medium than of the control were needed to inhibit the activity of $1 \mathrm{~mL}$ of the DPPH radical by $50 \%$. In the absence of E. sativa, L. acidophilus failed to decrease the volume of broth needed to lead to a $50 \%$ decrease in the activity of $1 \mathrm{~mL}$ of DPPH: EC50 increased from 74.73 at time zero to $92.13 \mu \mathrm{L}$ after $24 \mathrm{~h}$. In the presence of E. sativa, L. acidophilus did not affect the initial antioxidant activity. Indeed, the antioxidant power exhibited by its pellet increased by $5 \%$ if compared to the standard growth conditions, demonstrating that the presence of the vegetable in the culture medium and the concurrent growth of this strain did not give a lowering of the antioxidant activity of the medium; indeed, such a situation might beneficially impact the antioxidant activity of the strain, which could bring further positive effects, such as higher antioxidant activity, when grown under these conditions (for example, in the presence of fruits and vegetables) and then could be an additional means to counteract the action of free radicals. The presence of $L$. plantarum in the medium containing E. sativa mitigated, albeit more weakly than $L$. acidophilus, the loss of the antioxidant activity of the medium itself $\left(\mathrm{EC}_{50}=63 \mu \mathrm{L}\right)$. Furthermore, in this case, the presence of E. sativa gave rise to an increase of the anti-radical properties of the strain, which pellet increased its antioxidant power from $19 \%$ to approximately $24 \%$, in line with that exhibited by $L$. acidophilus. A quite different behavior was exhibited by L. rhamnosus. Although its presence did not affect the antioxidant activity of medium containing E. sativa, the strain was not positively affected by its own antioxidant activity; thus, after $24 \mathrm{~h}$ of growth, the percentage remained virtually the same (14\%) as that observed in standard conditions of growth (15\%). This could support the idea that bacteria have a metabolism that can be expressed in different ways and in a different manners depending on the presence of diverse components [20].

\subsection{Antimicrobial Activity}

Phytochemicals are able to change some beneficial probiotic species while inhibiting the growth of non-beneficial species, such as Bacteroides, Clostridia, Coliforms and Salmonella spp. [27,28]. The antimicrobial power exhibited by the three Lactobacillus after $24 \mathrm{~h}$ of growth, before and after the digestive passage, was measured using the inhibition halo test. The results are shown in Table 4 . In Table 5, pathogen sensitivity to DMSO, MRS, MRS + E. sativa and tetracycline are shown.

The presence of polyphenols in the culture medium might have transformed bacterial metabolism leading also to the production of substances with antimicrobial activity. L. plantarum grown in MRS demonstrated antimicrobial activity against all pathogenic strains used as standards, with halos of inhibition ranging between 2 and $12 \mathrm{~mm}$ (Table 4, green). In some case, the simulation of the gastro-intestinal transit demonstrated a slight decrease in the antimicrobial ability of the strain grown in MRS, in particular against E. coli (with a halo of inhibition of only $3 \mathrm{~mm}$, with the highest volume of culture supernatant used in our experiment, compared to a halo of $9 \mathrm{~mm}$, observed in the pre-gastric situation). The two strains of $B$. cereus used as standards showed slightly different behaviors, mainly after the simulated digestion. This suggests that for antioxidant activity, the presence of phytochemicals might affect the reaction of the microorganisms, even between two strains of the same species. For this reason, testing multiple strains of the same species in the evaluation of antimicrobial activities of any biological matrix (cells or natural extracts) should be considered. De Martino et al. [29], for example, 
observed a different behavior between two strains of $B$. cereus placed in contact with different essential oils and noted that $B$. cereus 4384 was more sensitive to the action of the essential oils tested than B. cereus 4313. These results, despite being obtained with Lamiaceae and not with Brassicaceae, are in agreement with our data. The presence of polyphenols in the growth medium caused a change in the behavior of $L$. plantarum compared with the control, especially after the simulation of the digestive process. L. acidophilus grown in MRS showed antimicrobial activity against all pathogens used as tester strains (Table 4, orange). The digestive process brought about a decrease of its antimicrobial activity, so that only $P$. aeruginosa retained a certain sensitivity (albeit decreased by $50 \%$ before in vitro digestion) at all three concentrations used in the experiment, and $B$. cereus was sensitive at the highest concentration used in our experiments. The presence of E. sativa increased the antimicrobial activity of $L$. acidophilus (the effect was enhanced after the simulated digestion) against $B$. cereus 4313 and S. aureus (with the highest concentration).

The antimicrobial activity of L. rhamnosus decreased (Table 4, purple), in some cases by more than $50 \%$ after in vitro digestion, but the presence of $E$. sativa did not deteriorate the antimicrobial activity of the strain, especially after simulated digestion. A $75 \%$ loss of activity in the pre-digestive step (mainly against Ps. aeruginosa and E. coli) was detected.

\subsection{Protein Profile}

The analysis of the protein profile of the three lactobacilli grown in the presence and absence of extracts of E. sativa was performed using on-chip microelectrophoresis. The results are shown in Figures 1-3. L. acidophilus (Figure 1) showed the greatest differences in protein patterns before the digestive in vitro process and with the presence or absence of $E$. sativa in the culture broth.

The two electropherograms (Figure 1A) showed large differences in protein profiles in the area ranging between $22 \mathrm{~s}$ and $37 \mathrm{~s}$. There was an evident flattening of the peaks in the representative proteins exhibited by the strain grown in MRS in this zone. In contrast, when the L. acidophilus strain was grown in the presence of E. sativa, the same area showed more peaks with molecular weights ranging from 8.3 to $78.72 \mathrm{kDa}$, in particular between 24.6 and $78.7 \mathrm{kDa}$. An MW of $39.29 \mathrm{kDa}$ represented $21.8 \%$ of the total proteins, and $46.93 \mathrm{kDa}$ represented $19.5 \%$ of the total proteins; these were the two most common proteins in the culture despite being undetectable in the control. After the simulated digestion, the two profiles were practically similar. A protein with an MW of $97.66 \mathrm{kDa}$ (marked with a circle) was observed only in the profile of the strain grown in the presence of $E$. sativa (Figure 1B), in addition to heavier proteins, MW $165 \mathrm{kDa}$ and $180 \mathrm{kDa}$, present only in traces. 
Table 4. Antimicrobial activity (evaluated through the inhibition halo test) of the filtered supernatant of MRS and MRS + E. sativa after the growth of L. acidophilus (LA, orange), L. plantarum (LP, green) and L. rhamnosus (LR, purple) before (BGPJ) and after (AGPJ) the in vitro gastro-intestinal transit. The results are reported as $\mathrm{mm}$ of halo ( \pm standard deviation) and are the mean of three independent experiments. BC 4313: Bacillus cereus 4313; BC 4384: Bacillus cereus 4384; SA: Staphylococcus aureus; EC: Escherichia coli; PA: Pseudomonas aeruginosa.

\begin{tabular}{|c|c|c|c|c|c|c|c|c|c|c|c|c|}
\hline & \multicolumn{2}{|c|}{ LP } & \multicolumn{2}{|c|}{$\mathbf{L P}+\mathbf{E}$} & \multicolumn{2}{|c|}{ LA } & \multicolumn{2}{|c|}{$\mathbf{L A}+\mathbf{E}$} & \multicolumn{2}{|c|}{ LR } & \multicolumn{2}{|c|}{$\mathbf{L R}+\mathbf{E}$} \\
\hline & BGPJ & AGPJ & BGPJ & AGPJ & BGPJ & AGPJ & BGPJ & AGPJ & BGPJ & AGPJ & BGPJ & AGPJ \\
\hline \multicolumn{13}{|c|}{ ВC 4313} \\
\hline $5 \mu \mathrm{L}$ & - & - & $5(0.5)$ & $2(0.3)$ & $3.4(0.3)$ & - & $3(0)$ & - & $5(0.3)$ & $1.2(0)$ & $2(0)$ & $2(0)$ \\
\hline $10 \mu \mathrm{L}$ & $4(0.3)$ & - & $7(0.3)$ & $4(0.2)$ & $6.3(0.3)$ & $3(0.3)$ & $6(0.1)$ & $4(0)$ & $7(0.5)$ & $3(0)$ & $6(0.1)$ & $3(0)$ \\
\hline $20 \mu \mathrm{L}$ & $8(0.8)$ & $10(0.4)$ & $12(0.2)$ & $6(0.3)$ & $10.3(0.3)$ & $2.5(0.5)$ & $7(0.6)$ & $10.5(0.3)$ & $12(0.7)$ & $5(0.5)$ & $10(0.4)$ & $6(0.2)$ \\
\hline \multicolumn{13}{|c|}{ ВС 4384} \\
\hline $5 \mu \mathrm{L}$ & $2.5(0.1)$ & $3(0.1)$ & $5(0.5)$ & $4(0.3)$ & $4(0)$ & - & $5(0.2)$ & $2(0)$ & $4(0.3)$ & $2(0.2)$ & $3.2(0.3)$ & $3(0.3)$ \\
\hline $10 \mu \mathrm{L}$ & $7(0.3)$ & $9(0.3)$ & $6(0.4)$ & $6(0.2)$ & $7(0.3)$ & $3(0)$ & $6.5(0.5)$ & $4(0.1)$ & $7.3(0.4)$ & $4(0.4)$ & $7.1(0.4)$ & $3(0.3)$ \\
\hline $20 \mu \mathrm{L}$ & $12(0.7)$ & $12(0.4)$ & $11(0.4)$ & $12(0.4)$ & $9.4(0.6)$ & - & $10(0.3)$ & $10(0.3)$ & $11(0.5)$ & $6(0.1)$ & $11.3(0.3)$ & $5.9(0.3)$ \\
\hline \multicolumn{13}{|c|}{$S A$} \\
\hline $5 \mu \mathrm{L}$ & - & - & $5(0.5)$ & $2(0.2)$ & $4.5(0.5)$ & - & $3.5(0.5)$ & $3(0.3)$ & $5(0)$ & $2(0)$ & $4(0.3)$ & $1.5(0)$ \\
\hline $10 \mu \mathrm{L}$ & $2(0.2)$ & $6(0.2)$ & $6(0.1)$ & $3(0.3)$ & $6.5(0.5)$ & $3(3.1)$ & $6.5(0.3)$ & $5(0.4)$ & $7(0.4)$ & $3(0)$ & $6(0.2)$ & $3(0)$ \\
\hline $20 \mu \mathrm{L}$ & $7(0.4)$ & $6(0.3)$ & $10(0.3)$ & $4(0.2)$ & $8.5(0.5)$ & $6(0.2)$ & $7.8(0.3)$ & $10(0.3)$ & $9(0.3)$ & $5(0.5)$ & $9(0.3)$ & $5(0.5)$ \\
\hline \multicolumn{13}{|c|}{$P A$} \\
\hline $5 \mu \mathrm{L}$ & $2(0.2)$ & $2(0.1)$ & $4(0.4)$ & $2(0.1)$ & $4(0.3)$ & $1.5(0.3)$ & $4.5(0.5)$ & - & - & - & $2.2(0.3)$ & - \\
\hline $10 \mu \mathrm{L}$ & $5(0.1)$ & $3(0.2)$ & $7(0.4)$ & $3(0.3)$ & $8(0.4)$ & $4(0.2)$ & $6(0.6)$ & $2(0.1)$ & $7(0.5)$ & $2(0.1)$ & $6(0.4)$ & $2(0.3)$ \\
\hline $20 \mu \mathrm{L}$ & $10(0.3)$ & $5(0.2)$ & $12(0.8)$ & $3(0.2)$ & $8.5(0.5)$ & $4(0.1)$ & $9(0.3)$ & $2(0.1)$ & $11(0.4)$ & $3(0.1)$ & $10.5(0.3)$ & $3.2(0.3)$ \\
\hline \multicolumn{13}{|c|}{$E C$} \\
\hline $5 \mu \mathrm{L}$ & - & - & $5(0.2)$ & $2(0.1)$ & $2.5(0.1)$ & - & $4.8(0.3)$ & $2(0.2)$ & $3(0.1)$ & $2(0)$ & - & - \\
\hline $10 \mu \mathrm{L}$ & $2(0.1)$ & - & $8(0.3)$ & $1(0)$ & $7(0.4)$ & $3(0.5)$ & $6.5(0.5)$ & $4(0.3)$ & $6(0.2)$ & $1.3(0)$ & $7.5(0.3)$ & - \\
\hline $20 \mu \mathrm{L}$ & $9(0.8)$ & $3(0.3)$ & $12(0.3)$ & $3(0)$ & $10.4(0.1)$ & $6(0.1)$ & $10(0.3)$ & $10(0.6)$ & $11(0.4)$ & $5(0)$ & $11(0.4)$ & $3(0)$ \\
\hline
\end{tabular}


Table 5. Antimicrobial activity (evaluated through the inhibition halo test) of the filtered supernatant of MRS and MRS + E. sativa, DMSO (negative control) and tetracycline (7 $\mu \mathrm{g}$, positive control) against Bacillus cereus 4313, Bacillus cereus 4384, Staphylococcus aureus, Escherichia coli and Pseudomonas aeruginosa.

\begin{tabular}{cccccccc}
\hline & \multicolumn{2}{c}{ Gram-positive tester strains } & & \multicolumn{3}{c}{ Gram-negative tester strains } \\
\cline { 2 - 3 } & P. aeruginosa & E. coli & & S. aureus & B. cereus 4313 & B. cereus 4384 \\
\hline DMSO & 0 & 0 & & 0 & 0 & 0 \\
Tetracycline $(7 \mu \mathrm{g})$ & $9.8(1.6)$ & $12(1.2)$ & & $11(0.4)$ & $9(0.6)$ & $8.4(1.4)$ \\
MRS & 0 & 0 & & 0 & 0 & 0 \\
MRS + E. sativa & 0 & 0 & & 0 & 0 & 0 \\
\hline
\end{tabular}

Figure 1. Protein profile (shown as an electropherogram) of Lactobacillus acidophilus, grown in MRS and MRS + E. sativa, before (LA PRE and LA + R PRE) (A) and after (LA POST and LA + R POST) (B) the in vitro gastro-intestinal transit.

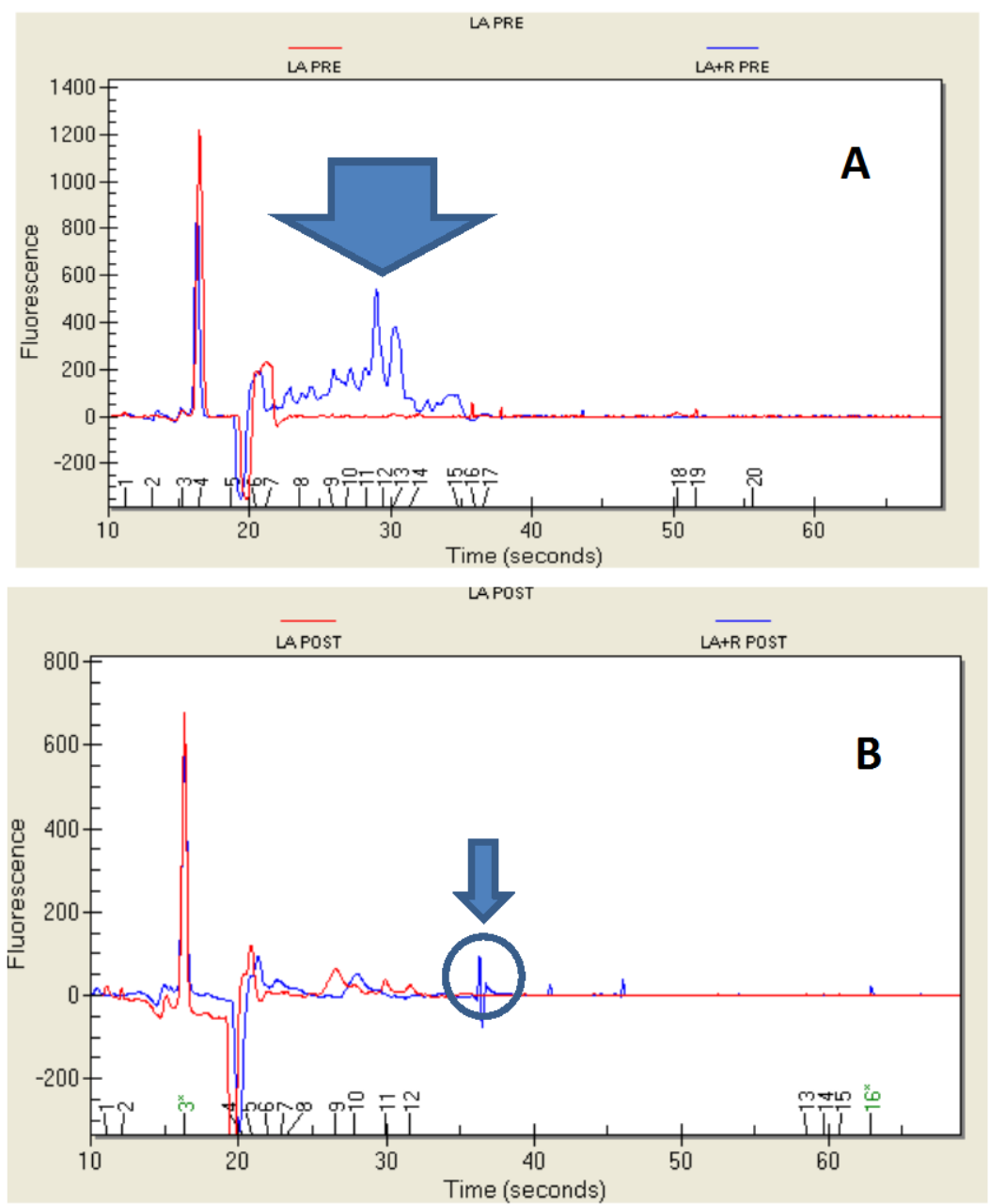

L. plantarum exhibited proteins with an MW ranging between 9.4 and $105.66 \mathrm{kDa}$ (Figure 2A). Several proteins were clear. Three of them had similar MWs (31.09 kDa, $32.3 \mathrm{kDa}$ and $33.74 \mathrm{kDa}$ ); a fourth had an MW of $79.06 \mathrm{kDa}$, and a fifth protein had an MW of $180.8 \mathrm{kDa}$. The fifth protein was absent after growth in the presence of E. sativa, but in this last case, a protein at MW $70.6 \mathrm{kDa}$ was observed that was absent in the control. When the strain was subjected to the simulated digestion, 
(Figure 2B), it showed three abundant proteins: MW $35.95 \mathrm{kDa}$ (43\% of the total), MW $58.43 \mathrm{kDa}$ (12\% of the total) and MW $71.4 \mathrm{kDa}$ (12\% of the total). The profile was slightly different when the strain grown in the presence of E. sativa was subjected to the simulated digestion. The first part of the electropherogram figure was more delineated than the control, and two distinct peaks were clearly visible. However, these were signals of the analytical system and were not taken into consideration. In the next portion of the electropherogram, eluted around $27 \mathrm{~s}$, two enlarged peaks were quite evident at MW $29.38 \mathrm{kDa}(60 \%$ of the total) and $36.78 \mathrm{kDa}(12.9 \%$ of the total).

Figure 2. Protein profile (shown as an electropherogram) of Lactobacillus plantarum, grown in MRS and MRS + E. sativa, before (LP PRE and LP + R PRE) (A) and after (LP POST and LP + R POST) (B) the in vitro gastro-intestinal transit.

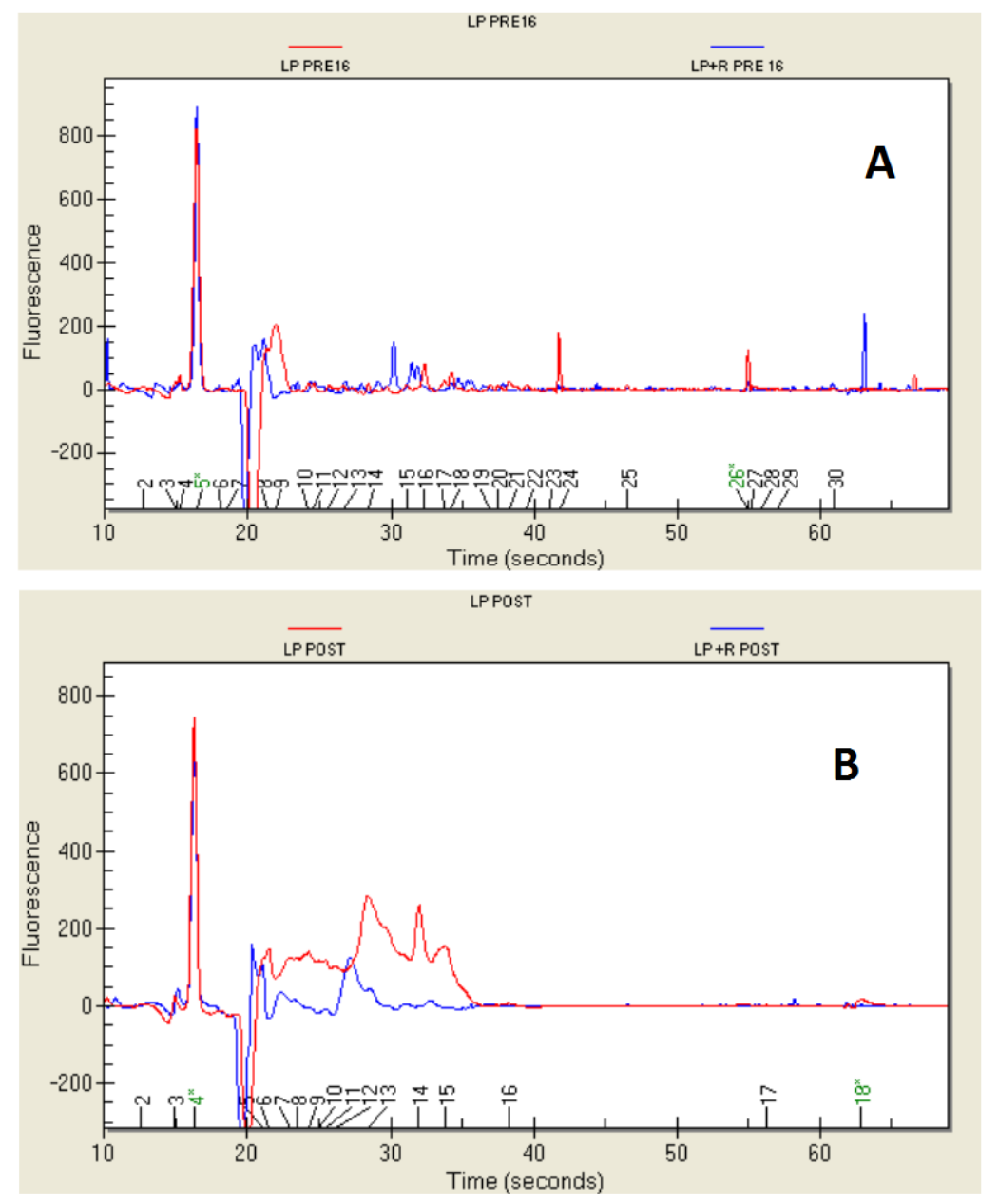

The proteins of $L$. rhamnosus grown in MRS ranged between 13 and $91 \mathrm{kDa}$ (Figure 3A). Two proteins were particularly representative: MW $23.85 \mathrm{kDa}$ (29\% of the total proteins) and $90.45 \mathrm{kDa}$ (21\% of the total proteins). In the simulated pre-gastric phase (indicated as PRE in the figure), two different proteins, MW $63.56 \mathrm{kDa}$ (11\% of the total) and MW $92 \mathrm{kDa}$ (73\% of the total), were detected in the control. The presence of E. sativa most likely affected the microbial protein profile; the strain showed proteins at an MW ranging between 53.76 and $122.14 \mathrm{kDa}$. After simulation of the gastro-intestinal transit, many proteins were detected in the control at MWs ranging from 9.3 to $127 \mathrm{kDa}$ (Figure 3B). The proteins at MW $9.42 \mathrm{kDa}$ and at MW $30 \mathrm{kDa}$ were the most abundant and represented $10.8 \%$ and 
$67 \%$ of the total protein pattern, respectively. In contrast, two proteins were observed after the growth of the strain in the presence of E. sativa and following the in vitro digestion at MW $9.30 \mathrm{kDa}$ (58.8\% of total) and at MW $51.17 \mathrm{kDa}(20.11 \%$ of total).

Figure 3. Protein profile (shown as electropherogram) of Lactobacillus rhamnosus grown in MRS and MRS + E. sativa before (LR PRE and LR + R PRE) (A) and after (LR POST and LR + R POST) (B) intestinal transit.

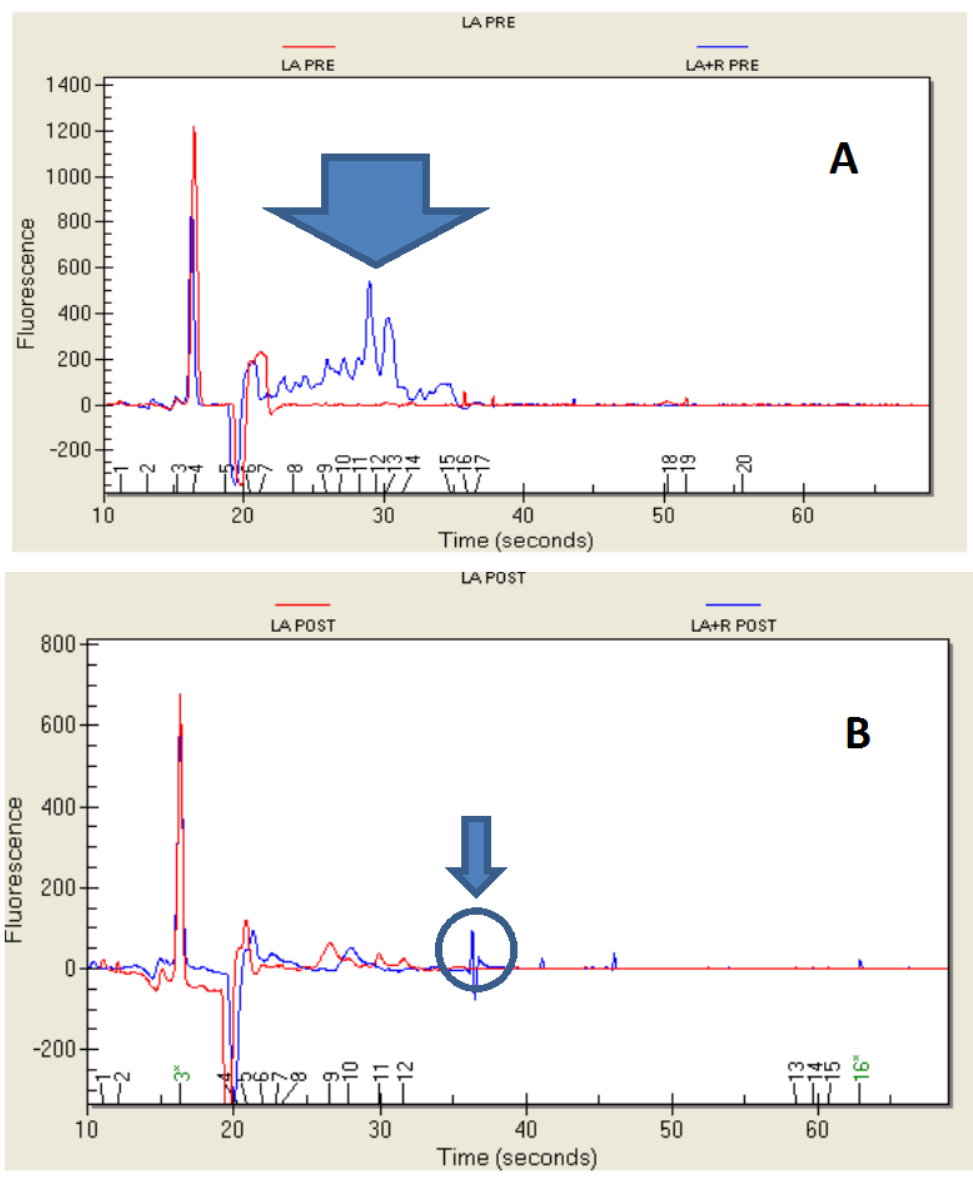

\section{Experimental Section}

\subsection{Strain and Culture Conditions}

Lactobacillus plantarum subsp. plantarum DSM 20174, Lactobacillus acidophilus DSM 20079, and Lactobacillus rhamnosus DSM 20711 are three DSMZ reference strains (Deutsche Sammlung von Mikroorganismen und Zellkulturen GmbH, Braunschweig, DSMZ, Germany). Cells were anaerobically grown at $30{ }^{\circ} \mathrm{C}$ (L. plantarum) and at $37{ }^{\circ} \mathrm{C}$ (L. acidophilus and L. rhamnosus) for $24 \mathrm{~h}$ in MRS (de Man, Rogosa and Sharpe) broth purchased by Sigma Aldrich Italia (Milano, Italy) [30]. Gram-positive Bacillus cereus (strains DSM 4313 and DSM 4384) and Staphylococcus aureus DSM 25923 and Gram-negative Escherichia coli DSM 8579 and Pseudomonas aeruginosa ATCC 50071 were the pathogenic strains used for the antimicrobial test; they were obtained from the DSMZ. Each strain was incubated at $37^{\circ} \mathrm{C}$ for $18 \mathrm{~h}$ in tryptone yeast extract (Oxoid, Milano, Italy). 


\subsection{Preparation of MRS Broth + Eruca sativa}

To make the medium, $100 \mathrm{~mL}$ of MRS broth was mixed with $100 \mathrm{~g}$ of E. sativa (purchased at a local market in Avellino, Italy; previously cleaned and cut into small pieces). The mixture was immediately treated with 5 cycles of microwaves for 20 s/each, using a domestic microwave oven (Candy J51, purchased at a local household store) at the maximum power (indicated as "10" by the apparatus). After $1 \mathrm{~h}$ at $4{ }^{\circ} \mathrm{C}$ in the dark, the mixture was treated again with microwaves (at the above conditions), held for $1 \mathrm{~h}$ at $4{ }^{\circ} \mathrm{C}$ and filtered $(0.22 \mu \mathrm{m}$, Millipore SpA, Milano, Italy). Aliquots of MRS + E. sativa and MRS alone (control) were inoculated with the three Lactobacillus and cultivated for $24 \mathrm{~h}$ in anaerobic conditions.

\subsection{Resistance to Simulated Gastric and Intestinal Juices}

The artificial gastric juice contained MRS broth plus 3 g/L pepsin (Sigma Aldrich Italia, Milano, Italy), with a final $\mathrm{pH}$ adjusted to 2.0 with $\mathrm{HCl}$ (Carlo Erba, Milano, Italy). The artificial intestinal juice contained MRS (Sigma Aldrich Italia, Milano, Italy) broth plus $1 \mathrm{~g} / \mathrm{L}$ pancreatin (Sigma Aldrich Italia, Milano, Italy) and $4.5 \mathrm{~g} / \mathrm{L}$ bile salts (sodium glycocholate and sodium taurocholate, code number X606C, Oxoid, Basingstoke, UK). Both solutions were filter-sterilized (0.22 $\mu \mathrm{m}$, Millipore SpA, Milano, Italy). The strain was treated using the protocols described by De Giulio et al. [31]. Cells were harvested by centrifugation for $5 \mathrm{~min}$ at $5000 \times \mathrm{g}$ at $4{ }^{\circ} \mathrm{C}$ (Biofuge, Beckmann Coulter Italia, Cassina de Pecchi, Italy), washed twice with physiological solution (0.85\% $\mathrm{NaCl}$, Carlo Erba, Milano, Italy) and centrifuged again. The pellet obtained was incubated in artificial gastric juice for $180 \mathrm{~min}$ at $37^{\circ} \mathrm{C}$ and recovered by centrifugation. After washing with sterile physiological solution, the pellet was re-suspended in the simulated gastrointestinal juice $(4.5 \mathrm{~mL}$ for $100 \mu \mathrm{L}$ of cells). Samples were incubated at $37{ }^{\circ} \mathrm{C}$ for $60 \mathrm{~min}$. Cell viability was evaluated by anaerobic culturing on MRS plates before and after the incubation of the strains in the two simulated gastrointestinal juices. Each separate experiment was performed three times.

\subsection{Colorimetric Analysis of Polyphenols}

The total phenolic content was determined using the Folin-Ciocalteu method as described by Singleton and Rossi [32]. Then, $750 \mu \mathrm{L}$ of pure water and $50 \mu \mathrm{L}$ of the Folin-Ciocalteu reagent (Bio-Rad, Milano, Italy) were added to $50 \mu \mathrm{L}$ of the suitably diluted sample extract. The mixture was held for $6 \mathrm{~min}$, then $100 \mu \mathrm{L}$ of a 7\% aqueous $\mathrm{Na}_{2} \mathrm{CO}_{3}$ (Sigma-Aldrich Italia, Milano, Italy) solution was added. After $120 \mathrm{~min}$, the absorption was measured at $760 \mathrm{~nm}$ against water as a blank, using a Cary UV/Vis spectrophotometer (Varian, Palo Alto, CA, USA). The amount of total phenolics was expressed as $\mu \mathrm{g}$ gallic acid equivalents (GAE)/mL of extract.

\subsection{Preparation of Heat Killed Cells}

Preparation of heat killed cells (HKC) was performed following the method described by Liu et al. [33] and Nazzaro et al. [34], with some modifications. Cells were harvested by centrifugation for $5 \mathrm{~min}$ at $5000 \times \mathrm{g}$ at $4{ }^{\circ} \mathrm{C}$ (Biofuge, Beckmann Coulter Italia, Cassina de Pecchi, Italy). The pellet was washed twice and re-suspended in phosphate buffered saline (pH 7.2) buffer (Carlo Erba, Milano, 
Italy), heated at $100{ }^{\circ} \mathrm{C}$ for $30 \mathrm{~min}$ and dried. Samples were re-suspended again at $5 \mathrm{mg}$ dry cell/mL, autoclaved at $121{ }^{\circ} \mathrm{C}$ for $15 \mathrm{~min}$ and stored in $4{ }^{\circ} \mathrm{C}$ until the analysis of the radical scavenging capacity was performed.

\subsection{Free Radical Scavenging Capacity}

The free radical scavenging capacity was determined using the stable radical 2,2-diphenyl-1-picrylhydrazyl (DPPH) assay [35]. The analysis was performed in microplates by adding $7.5 \mu \mathrm{L}$ of extract to $303 \mu \mathrm{L}$ of a methanol DPPH solution (153 mM, handmade starting from DPPH (Sigma-Aldrich Italia, Milano, Italy) and methanol (Carlo Erba, Milano, Italy)). Next, the absorbance at $\lambda=517 \mathrm{~nm}$ was measured (Cary $50 \mathrm{MPR}$, Varian, Palo Alto, USA). The absorbance of DPPH without antioxidants (control sample) was used for baseline measurements. The scavenging activity was expressed as the $50 \%$ effective concentration (EC50), which was defined as the sample volume $(\mu \mathrm{L})$ necessary to inhibit $1 \mathrm{~mL}$ of DPPH radical activity by $50 \%$ during a 60 -min incubation. These experiments were performed in triplicate, and the results are expressed as the mean values \pm standard deviations.

\subsection{Protein Profile}

Cells were centrifuged ( $5 \mathrm{~min}$ at $5000 \times \mathrm{g}$ at $4{ }^{\circ} \mathrm{C}$, Biofuge, Beckmann Coulter Italia, Cassina de Pecchi, Italy) and washed twice in $0.05 \mathrm{M}$ Tris- $\mathrm{HCl}$, pH 7.4 (1:5 w:v, handmade from Trizma base (Sigma-Aldrich Italia, Milano, Italy) and $\mathrm{HCl}$ (Carlo Erba, Milano, Italy). Pellets were re-suspended in $300 \mu \mathrm{L} 0.05 \mathrm{M}$ Tris-HCl, pH 7.4, with 2\% SDS. Following the addition of glass beads, samples were constantly mixed for $5 \mathrm{~min}$ and then treated for $3 \mathrm{~min}$ at $100{ }^{\circ} \mathrm{C}[34,36]$. Glass beads and cell debris were removed by centrifugation, and the supernatant was recovered. The protein content was evaluated according to the method reported by Bradford [37]. A 5- $\mu \mathrm{L}$ aliquot of each sample was diluted with $84 \mu \mathrm{L}$ of ultrapure water, and then, $2 \mu \mathrm{L}$ of sample buffer (Experion ${ }^{\mathrm{TM}}$ Pro260 kit, Bio-Rad, Hercules, CA, USA) containing $1 \mu \mathrm{L}$ of $\beta$-mercaptoethanol (Sigma-Aldrich Italia, Milano, Italy) were added. All samples were treated at $100{ }^{\circ} \mathrm{C}$ for $3 \mathrm{~min}$. A $6-\mu \mathrm{L}$ sample was analyzed via chip capillary electrophoresis (Experion ${ }^{\mathrm{TM}}$ Pro260 Analysis Kit, Hercules, CA, USA) over a range of molecular weights from 1.2 to $260 \mathrm{kDa}$. The analysis was completed using an Experion ${ }^{\mathrm{TM}}$ automated electrophoresis system (Bio-Rad Hercules, CA, USA) and the appropriate software (fluorescence detection with a 10-mW semiconductor, laser emitting at $630 \mathrm{~nm}$ ). The automated analysis required $30 \mathrm{~min}$ for a set of 10 samples.

\subsection{Antimicrobial Assays}

The inhibition halo test on agar plates was employed to investigate the antibacterial activity of the lactobacilli before and after the simulated digestive process, and the filter paper disc method was used. Microbial suspensions $\left(1 \times 10^{8}\right.$ colony forming units-CFU-/mL) were uniformly spread onto the tryptone yeast extract agar plates ( $\varnothing=90 \mathrm{~mm}$ dishes). Sterile Whatman No. 1 paper filter discs $(\varnothing=5 \mathrm{~mm})$ were impregnated with $5 \mu \mathrm{L}, 10 \mu \mathrm{L}$ and $20 \mu \mathrm{L}$ of the medium of MRS and MRS + E. sativa recovered after the growth of lactobacilli and filtered. Tetracycline (7 $\mu \mathrm{g} /$ disc; Sigma-Aldrich Italia, Milano, Italy) was used as positive control. DMSO (10 $\mu \mathrm{L}$ /paper disc Sigma-Aldrich Italia, Milano, Italy), MRS and 
MRS + E. sativa were used as negative controls. Plates were left for $30 \mathrm{~min}$ at room temperature under sterile conditions and then incubated at $37^{\circ} \mathrm{C}$ for $24 \mathrm{~h}$, after which the inhibition halo around the disc was measured. The experiments were performed in triplicate and averaged.

\section{Conclusions}

The presence of E. sativa affected some of the biological properties of the three lactobacilli. Notably, in most cases, they increased both the antioxidant activity of the medium and their own antioxidant power, enhancing one of the essential characteristics of probiotic bacteria. For example, L. acidophilus increased the consumption of polyphenols present in the medium, with a concurrent increases in the antioxidant power of the strain and in the resistance to the simulated digestive passage. L. plantarum increased its capability to inhibit the activity of the DPPH radical and increased its growth, albeit to a lesser extent than $L$ acidophilus. The behavior of $L$. rhamnosus was very different: It did not seem to "accept" the presence of E. sativa in the medium, reducing both growth and antioxidant activity. With regard to the analysis of proteins, the most visible changes were observed in L. acidophilus with an enhanced protein profile compared with the control. This suggests that at least in the pre-digestive step, the synthesis of particular proteins by this Lactobacillus, grown in the presence of polyphenols, could support the improvement of its biological properties. In contrast, the simulated digestive process resulted in a flattening of differences in protein profiles so that differences in antimicrobial activity could not be ascribed to the expression of different proteins. The protein profile of L. plantarum showed some changes found both before and after the simulated gastro-intestinal transit compared with the control; however, such differences did not seem to influence its antimicrobial activity. L. rhamnosus showed only slight differences, which were practically annulled after the in vitro digestion (which did not have an obvious influence on the activity of the microbial strain). Overall, the presence of vegetables might help boost some of the characteristics of lactobacilli, including the antioxidant and antimicrobial power.

\section{Acknowledgments}

The work was supported by the PON Smart Cities and Communities and Social Innovation (project “ABSIDE”, subsystem "BE\&SAVE”, Code No. PON04a2_F) of the Italian Ministry of University, Instruction and Scientific Research.

\section{Author Contributions}

Florinda Fratianni participated in the design of the experimental plan, in the analysis of data and the discussion of the results. Selenia Pepe, Federica Cardinale, Autilia Cozzolino and Tiziana Granese participated in the experimental phase. Raffaele Coppola was co-responsible for the research activity of ISA-CNR for the project “ABSIDE” (subsystem "BE\&SAVE”) and participated in the planning of the experiments, as well as in the analysis and discussion of data. Filomena Nazzaro was co-responsible for the research activity of ISA CNR for the project “ABSIDE” (subsystem "BE\&SAVE”), designed the experimental plans, analyzed and discussed the experimental data analysis and wrote the paper. All authors read and approved the final manuscript. 


\section{Conflicts of Interest}

The authors declare no conflict of interest.

\section{References}

1. Block, G.; Patterson, B.; Subar, A. Fruit, vegetables and cancer prevention. A review of the epidemiological evidence. Nutr. Cancer 1992, 18, 1-29.

2. Halliwell, B. Protection against tissue damage in vivo by desferrioxammine: What is its mechanism of action? Free Rad. Biol. Med. 1989, 7, 645-651.

3. Hertog, M.G.L.; Freskens, E.J.M.; Hollman, P.C.H.; Katan, M.B.; Kromhout, D. Dietary antioxidant flavonoids and the risk of coronary heart disease: The zutphen elverly study. Lancet 1993, 342, 1007-1011.

4. Heim, K.E.; Tagliaferro, A.R.; Bobilya, D.J. Flavonoid antioxidants: Chemistry, metabolism and structure-activity relationships. J. Nutr. Biochem. 2002, 13, 572-584.

5. Higdon, J.V.; Delage, B.; Williams, D.E.; Dashwood, R.H. Cruciferous vegetables and human cancer risk: Epidemiologic evidence and mechanistic basis. Pharm. Res. 2007, 55, 224-236.

6. Jin, J.; Koroleva, O.A.; Gibson, T.; Swanston, J.; Magan, J.; Zhang, Y.; Rowland, I.A.; Wagstaff, C. Analysis of phytochemical composition and chemoprotective capacity of rocket (Eruca sativa and Diplotaxis tenuifolia) leafy salad following cultivation in different environments. J. Agric. Food Chem. 2009, 57, 5227-5234.

7. Selma, M.V; Espín, J.C.; Tomás-Barberán, F.A. Interaction between phenolics and gut microbiota: Role in human health. J. Agric. Food Chem. 2009, 57, 6485-6501.

8. Wells, C.L.; Jechorek, R.P.; Kinneberg, K.M.; Debol, S.M.; Erlandsen, S.L. The isoflavone genistein inhibits internalization of enteric bacteria by cultured Caco-2 and HT-29 enterocytes. J. Nutr. 1999, 129, 634-640.

9. Parkar, S.G.; Stevenson, D.E.; Skinner, M.A. The potential influence of fruit polyphenols on colonic microflora and human gut health. Int. J. Food Microbiol. 2008, 124, 295-298.

10. Vattem, D.A.; Lin, Y.T.; Ghaedian, R.; Shetty, K. Cranberry synergies for dietary management of Helicobacter pylori infections. Process Biochem. 2005, 40, 1583-1592.

11. Rodríguez-Vaquero, M.J.; Alberto, M.R.; Manca de Nadra, M.C. Antibacterial effect of phenolic compounds from different wines. Food Control 2007, 18, 93-101.

12. Almajano, M.P.; Carbó, R.; López-Jiménez, J.A.; Gordon, M.H. Antioxidant and antimicrobial activities of tea infusions. Food Chem. 2008, 108, 55-63.

13. Nazzaro, F.; Fratianni, F.; Coppola, R. Quorum sensing and phytochemicals. Int. J. Mol. Sci. 2013, 14, 12607-12619.

14. Fratianni, F.; de Martino, L.; Melone, A.; de Feo, V.; Coppola, R.; Nazzaro, F. Preservation of chicken breast meat treated with thyme and balm essential oils. J. Food Sci. 2010, 75, M528-M535.

15. Rodríguez, H.; Curiel, J.A.; Landete, J.M.; de Las Rivas, B.; de Felipe, F.L.; Gómez-Cordovés, C.; Mancheño, J.M.; Muñoz, R. Food phenolics and lactic acid bacteria. Int. J. Food Microbiol. 2009, 132, 79-90. 
16. Hervert-Hernández, D.; Pintado, C.; Rotger, R.; Goñi, I. Stimulatory role of grape pomace polyphenols on Lactobacillus acidophilus growth. Int. J. Food Microbiol. 2009, 136, 119-122.

17. Fratianni, F.; Coppola, R.; Sada, A.; Mendiola, J.A.; Ibanez, E.; Nazzaro, F. A novel functional probiotic product containing phenolics and anthocyanins. Int. J. Probiotics Prebiotics 2010, 5, 85-90.

18. Fratianni, F.; Cardinale, F.; Russo, I.; Iuliano, C.; Tremonte, P.; Coppola, R.; Nazzaro, F. Ability of synbiotic encapsulated Saccharomyces cerevisiae boulardii to grow in berry juice and to survive under simulated gastrointestinal conditions. J. Microencapsul. 2013, 31, 299-305.

19. Fratianni, F.; Cardinale, F.; Russo, I.; Iuliano, C.; Cucciniello, A.C.; Maione, M.; d’Acierno, A.; Nazzaro, F. Fermentation of tomato juice with the probiotic yeast Saccharomyces cerevisiae boulardii. In Functional Foods: Sources, Biotechnology Applications, and Health Challenges; Robinson, A., Emerson, D., Eds.; Nova Science Publisher: New York, NY, USA, 2013; pp 143-152.

20. Nazzaro, F.; Fratianni, F.; Sada, A.; Orlando, P. Synbiotic potential of carrot juice supplemented with Lactobacillus spp. and inulin or fructooligosaccharides. J. Sci. Food Agric. 2008, 88, 2271-2276.

21. China, R.; Mukherjee, S.; Sen, S.; Bose, S.; Datta, S.; Koley, H.; Ghosh, S.; Dhar, P. Antimicrobial activity of Sesbania grandiflora flower polyphenol extracts on some pathogenic bacteria and growth stimulatory effect on the probiotic organism Lactobacillus acidophilus. Microbiol. Res. 2012, 167, 500-506.

22. Granato, D.; Branco, G.F.; Nazzaro, F.; Cruz, A.; Faria, J.A.F. Functional foods and nondairy probiotic food development: Trends, concepts, and products. Compr. Rev. Food Sci. Food Saf. 2010, 9, 292-302.

23. FAO/WHO Experts' Report, 2001 FAO/WHO Experts' Report. Processing of Health and Nutritional Properties of Probiotics in Food Including Powder Milk With Live Lactic Acid Bacteria, Córdoba, Argentina, 1-4 October 2001.

24. Laparra, J.M.; Sanz, Y. Interactions of gut microbiota with functional food components and nutraceuticals. Pharm. Res. 2010, 61, 219-225.

25. Irshad, M.; Ahmad, I.; Goel, H.C.; Rizvi, M.M.A. Phytochemical screening and high performance TLC analysis of some cucirbits. Res. J. Phytochem. 2010, 4, 242-247.

26. Sharma, D.; Rawat, I.; Goel, H.C. Antioxidant and prebiotic potential of some Cucurbits. Res. J. Med. Plant 2012, 6, 500-510.

27. Lee, H.C.; Jenner, A.M.; Lowand, C.S.; Lee, Y.K. Effect of tea phenolics and their aromatic fecal bacterial metabolites on intestinal microbiota. Res. Microbiol. 2006, 157, 876-884.

28. Chiva-Blancha, G.; Visioli, F. Polyphenols and health: Moving beyond antioxidants. J. Berry Res. 2012, 2, 63-71.

29. De Martino, L.; de Feo, V.; Nazzaro, F. Chemical composition and in vitro antimicrobial and mutagenic activities of seven Lamiaceae essential oils. Molecules 2009, 14, 4213-4230.

30. De Man, J.D.; Rogosa, M.; Sharpe, M.E. A medium for the cultivation of Lactobacilli. J. Appl. Bacteriol. 1960, 23,130-135.

31. De Giulio, B.; Orlando, P.; Barba, G.; Coppola, R.; de Rosa, M.; Sada, A.; de Prisco, P.P.; Nazzaro, F. Use of alginate and cryo-protective sugars to improve the viability of lactic acid bacteria after freezing and freeze-drying. World J. Microbiol. Biotechnol. 2005, 2, 739-748.

32. Singleton, V.L.; Rossi, J.A. Colorimetry of total phenolic with phosphomolybdic-phosphotungstic acid reagents. Am. J. Enol. Vitic. 1965, 16, 144-158. 
33. Liu, C.F.; Pan, T.M. In vitro effects of lactic acid bacteria on cancer cell viability and antioxidant activity. J. Food Drug Anal. 2010, 18, 77-86.

34. Nazzaro, F.; Fratianni, F.; Orlando, P.; Coppola, R. Biochemical traits, survival and biological properties of the probiotic Lactobacillus plantarum grown in the presence of prebiotic inulin and pectin as energy source. Pharmaceuticals 2012, 5, 481-492.

35. Brand-Williams, W.; Cuvelier, M.E.; Berset, C. Use of a free radical method to evaluate antioxidant activity. LWT-Food Sci. Technol. 1995, 28, 25-30.

36. Nazzaro, F.; Fratianni, F.; Nicolaus, B.; Poli, A.; Orlando, P. The prebiotic source influences the growth, biochemical features and survival under simulated gastrointestinal conditions of the probiotic Lactobacillus acidophilus. Anaerobe 2012, 18, 280-285.

37. Bradford, M.M. A rapid and sensitive method for the quantitation of microgram quantities of protein utilizing the principle of protein dye binding. Anal. Biochem. 1976, 72, 248-254.

(C) 2014 by the authors; licensee MDPI, Basel, Switzerland. This article is an open access article distributed under the terms and conditions of the Creative Commons Attribution license (http://creativecommons.org/licenses/by/4.0/). 